\title{
MECANISMOS ALTERNATIVOS PARA LA SOLUCIÓN DE CONTROVERSIAS EN EL CONTEXTO DE LOS DERECHOS EN SALUD: EXPERIENCIA PERUANA DESDE EL CENTRO DE CONCILIACION Y ARBITRAJE DE LA SUPERINTENDENCIA NACIONAL DE SALUD
}

\author{
Darwin Hidalgo-Salas ${ }^{1,2, a}$, Claudia Ortiz-Pérez ${ }^{3, b}$, Juan Lobatón-Flores ${ }^{4, c}$, Percy Huamaní-Ñahuinlla ${ }^{4, d}$, \\ Edward Mezones-Holguín ${ }^{4,5, \text { e }}$
}

\begin{abstract}
RESUMEN
En Perú se reconoce constitucionalmente el derecho a la protección de la salud; asimismo, la ley establece que la población tiene derecho al acceso a servicios de salud, a la información, a la atención y recuperación de su salud, al consentimiento informado y a la protección de sus derechos en salud. No obstante, con la ejecución de acciones relacionadas a ello, pueden presentarse inconformidades que requieren ser solucionadas. En ese marco, los mecanismos alternativos de solución de controversias (MASC) -como la mediación, la conciliación y el arbitraje- son vías válidas y accesibles en el Sistema de Salud Peruano. Actualmente, el Centro de Conciliación y Arbitraje de la Superintendencia Nacional de Salud (CECONAR) lidera la puesta en marcha de los MASC a nivel nacional. En este artículo se ofrece una visión general de CECONAR con un recorrido por sus bases históricas y legales, para luego mostrar su accionar presente $\mathrm{y}$, finalmente, discutir y delinear algunas perspectivas a futuro.
\end{abstract}

Palabras clave: Derecho a la salud; Solución de problemas; Sistemas de salud; Arbitraje; Rol judicial; Perú (Fuente: DECS BIREME).

\section{ALTERNATIVE MECHANISMS FOR CONFLICT RESOLUTION IN THE CONTEXT OF HEALTH RIGHTS: THE PERUVIAN EXPERIENCE FROM THE CENTER FOR RECONCILIATION AND ARBITRATION OF THE NATIONAL HEALTH AUTHORITY}

\begin{abstract}
In Peru the right to health protection is constitutionally recognized. The right to access health services is legally mandated, as well as access to health information and the protection of rights such as informed consent. However, with the implementation of actions related to these rights and protections, there may be disagreements which need to be resolved. In this context, alternative mechanisms for conflict resolution (AMCR), such as mediation, reconciliation and arbitration, are valid and accessible strategies within the Peruvian Health System. Currently, the Center for Reconciliation and Arbitration (CECONAR) of the National Health Authority (SUSALUD) has the responsibility to lead the implementation of AMCR at the national level. In this article we provide a general overview of CECONAR, starting with its historical and legal foundations, and continuing to its present activities and finally we discuss and outline some future prospects.
\end{abstract}

Key words: Right to health; Problem solving; Health systems; Arbitrating; Judicial role; Peru (Source: MeSH NLM).

\section{INTRODUCCIÓN}

Los derechos en salud (DES) son inherentes a la persona ${ }^{(1)}$ y su ejercicio pleno denota una serie de acciones por parte de los ciudadanos en el sistema de salud ${ }^{(2)}$. En Perú se reconoce constitucionalmente el derecho a la salud ${ }^{(3)}$, y se estipula por Ley que la población tiene derecho al acceso a servicios de salud, al acceso a la información, a la atención y recuperación de su salud, al consentimiento informado y a la protección de sus derechos ${ }^{(4)}$. Durante la puesta

\footnotetext{
Centro de Conciliación y Arbitraje, Superintendencia Nacional de Salud. Lima, Perú.

Facultad de Medicina Humana, Universidad Privada de San Martin de Porres. Lima, Perú.

Facultad de Derecho, Universidad de San Martín de Porres. Lima, Perú.

Intendencia de Investigación y Desarrollo, Superintendencia Nacional de Salud. Lima, Perú.

Escuela de Medicina, Universidad Peruana de Ciencias Aplicadas. Lima, Perú.

Abogado, máster en Derecho Sanitario y Bioética; ${ }^{\mathrm{b}}$ estudiante de Derecho; ${ }^{\mathrm{c}}$ licenciado en Computación; ${ }^{\mathrm{d}}$ licenciado en Estadística; ${ }^{\mathrm{e}}$ médico cirujano, maestro en Ciencias en Epidemiología Clínica.

Recibido: 14/05/2016 Aprobado: 15/06/2016
}

Citar como: Hidalgo-Salas D, Ortiz-Pérez C, Lobatón-Flores J, Huamaní-Ñahuinlla P, Mezones-Holguín E. Mecanismos alternativos para la solución de controversias en el contexto de los derechos en salud: experiencia peruana desde el Centro de Conciliación y Arbitraje de la Superintendencia Nacional de Salud. Rev Peru Med Exp Salud Publica. 2016;33(3)567-73. doi:10.17843/rpmesp.2016.333.2300 
en marcha de las distintas actividades vinculadas a estos derechos, pueden presentarse eventos que conlleven al surgimiento de controversias y conflictos que pueden involucrar usuarios, prestadores, financiadores, entre otros actores del sector (5-7); lo cual podría afectar negativamente el correcto desenvolvimiento del sistema de salud peruano (SSP) ${ }^{(8)}$. En consecuencia, resulta imperativo dotar de mecanismos alternativos de solución de controversias (MASC) que versen sobre los DES.

En Perú, la potestad de administrar justicia es ejercida por el Poder Judicial a través de sus órganos jerárquicos, sin embargo, existen estrategias alternativas para la solución de conflictos ${ }^{(3)}$. Si bien el proceso judicial - reconocido también como sistema adversarial- es el mecanismo más utilizado, la legislación faculta la posibilidad de someter determinadas 0 determinables controversias sobre derechos de libre disposición, mediante el empleo de mecanismos que permitan solucionarlos de manera más célere, efectiva y eficiente ${ }^{(9-11)}$. Estas vías conforman un conjunto de rutas útiles que permiten construir una solución propia en base a los intereses de las partes sustentados en el derecho.

Los MASC son canales válidos, no obstante, suelen ser desconocidas por la población, lo cual repercute en su bajo uso ${ }^{(12)}$. Dentro de los MASC se contemplan: la negociación o transacción, la mediación, la conciliación y el arbitraje ${ }^{(12-14)}$. La negociación es el intercambio de opiniones entre las partes de manera voluntaria, sin que para ello se requiera intermediación de un tercero, con la finalidad que las partes solucionen la controversia ${ }^{(12,15)}$. Por otro lado, la mediación requiere que un tercero imparcial acerque y facilite la comunicación entre las partes, proponiendo medios aceptables para que logren un acuerdo sin discrepancias, donde el mediador realiza la interposición de sus buenos oficios (IBOS), con el propósito de que sean ellas quienes decidan respecto al tema controvertido ${ }^{(12,14,15)}$. En el caso de la conciliación, las partes recurren a un tercero neutral que podrá hacer sugerencias de solución, para lo cual los convocará con el objeto de ayudar y facilitar el diálogo; sin embargo, serán únicamente las partes quienes voluntariamente definirán el resultado del conflicto ${ }^{(12,14,16)}$. Finalmente, es un método a través del cual un tercero imparcial impone su decisión frente a las partes y resuelve el conflicto por ellas, quienes de modo obligatorio o de manera previa, se han sometido voluntariamente a la decisión que emita el árbitro o tribunal arbitral; al respecto, dicha decisión se encontrará contenida en el laudo arbitral, el mismo que será vinculante para las partes ${ }^{(9,16,17)}$. Estos mecanismos tiene un alto potencial de uso en el área de la salud, ergo, el fomento de su utilización y la provisión de los mismos puede es una medida con alto impacto positivo en el SSP.
Por lo expuesto, y en virtud a la especial relevancia de las controversias suscitadas en el SSP, el Centro de Conciliación y Arbitraje (CECONAR) de la Superintendencia Nacional de Salud (SUSALUD) se ha convertido en una estrategia legítima de alta relevancia, al procurar la solución de dichas controversias, a través de la conciliación, mediación y arbitraje. El presente artículo brinda una descripción general de CECONAR, se parte de sus cimientos históricos y legales, para luego abordar el accionar en el presente $y$, finalmente, se delínea una discusión sobre el tópico y se esbozan lineamientos a futuro. Se considera que el aprovisionar de estos métodos alternativos accesibles, especializados, transparentes e imparciales $(12,14,16)$, contribuyen substancialmente a la mejora del sector Salud en Perú en el contexto de los DES.

\section{BASES HISTÓRICAS Y LEGALES}

Con la promulgación de la Ley de Modernización de la Seguridad Social en el año 1997 se forjan las bases para la creación del CECONAR. Esta iniciativa legal modificó la normativa del Instituto Peruano de Seguridad Social (IPSS) con la incorporación de las aseguradoras y servicios de salud privados en la seguridad social mediante las entidades prestadoras de salud (EPS), y la subsecuente creación de la Superintendencia de Entidades Prestadoras de Salud (SEPS) ${ }^{(18)}$. La SEPS, en virtud a que se le encarga emitir un reglamento de arbitraje y solución de controversias (19), erige el CECONAR, como un organismo autónomo que brinda servicios de conciliación y arbitraje en salud, y además asume competencias para resolver en vía arbitral, las controversias que surjan de la aplicación del seguro complementario de trabajo de riesgo (SCTR) ${ }^{(20,21)}$.

Posteriormente, en el año 2009 se promulgó la Ley Marco del Aseguramiento Universal en Salud (AUS), con lo cual se establecen canales de comunicación transaccionales entre los operadores de los seguros y servicios de salud públicos, privados y mixtos, de cara a alcanzar una intercambiabilidad plena para que el usuario acceda a todos los servicios de salud, independientemente del asegurador (22). En el marco del AUS, se funda la Superintendencia Nacional de Aseguramiento en Salud (SUNASA) sobre la base de la SEPS, la cual fue creada como un organismo público técnico especializado encargado de registrar, autorizar, supervisar y regular a las instituciones administradoras de fondos de aseguramiento en salud (IAFAS) y supervisar a las instituciones prestadoras de servicios de salud (IPRESS) ${ }^{(23)}$. Asimismo, se estipula que SUNASA debe instaurar estrategias de conciliación y arbitraje entre los usuarios, las IAFAS y las IPRESS vinculadas al proceso del AUS ${ }^{(23)}$. En el año 2010 se regula el funcionamiento del CECONAR otorgándosele autonomía funcional, asimismo, se concibe por 
primera vez un sistema nacional de conciliación y arbitraje en salud ${ }^{(24)}$. No obstante, más allá de algunas aproximaciones académicas y técnicas, CECONAR no logró desarrollar el mandato legal de proponer la regulación que regiría el aludido sistema.

En el marco de la reforma de la salud desarrollada a finales del 2013, se dictó el Decreto Legislativo 1158, donde se disponen medidas destinadas al fortalecimiento de las competencias de SUNASA, convirtiéndola en la Superintendencia Nacional de Salud (SUSALUD) ${ }^{(25)}$. Con ello se confiere -con rango de ley- al CECONAR autonomía técnica y funcional, y se le otorgan distintas competencias en materia de resolución de controversias en salud. Asimismo, se le otorga la potestad de administrar el servicio nacional de conciliación y arbitraje en salud (SENACOAS), lo que conlleva a desarrollar el registro y habilitación de los centros de conciliación y arbitraje privados con especialización en salud, con el objeto de establecer estándares, mecanismos de supervisión, fiscalización y otros; y subsecuentemente, a administrar el listado de conciliadores y árbitros adscritos a este sistema, bajo estándares y mecanismos de supervisión. Todo ello en la búsqueda de garantizar la idoneidad técnica, neutralidad, independencia y eficiencia en los procesos, que resulten en una disminución de las barreras de acceso a los MASC. También se concede al CECONAR la facultad de dirimir conflictos entre dos o más centros a los que se haya sometido una controversia, y actuar como centro competente cuando se haya acordado el sometimiento a arbitraje y no se alcance acuerdo sobre el centro al cual acudir. Adicionalmente, se autoriza al CECONAR, a implementar procedimientos para el auxilio económico de costos procesales para los usuarios que así lo requieran, cuando sea centro competente para la resolución de la controversia ${ }^{(25)}$.

\section{HACIA LA EXPANSIÓN, GRATUIDAD Y CONSOLIDACIÓN DE LOS SERVICIOS DEL CENTRO DE CONCILIACION Y ARBITRAJE}

Las cifras de las atenciones de CECONAR en IBOS, conciliación y arbitraje, sin la ampliación de los servicios, nos muestran un incremento en el tiempo, pese a que todos ellos fueron atendidos en la única oficina que CECONAR ha tenido desde su creación. En la Figura 1 se presenta un recuento histórico de la frecuencia de solicitudes de procesos en el lustro comprendido entre los años 2011 al 2015 en CECONAR, se evidencia una mayor frecuencia de solicitudes en el año 2015 en relación a los años previos; asimismo, se observa que en todos los años analizados el arbitraje fue el proceso más frecuente. En la Tabla 1 se advierte que la mayoría de arbitrajes son iniciados por los asegurados o beneficiados $(83,8 \%)$ frente a los empezados por las IAFAS (16,2\%). En ambos casos, la mayor parte de los procesos se dan en materia de seguro complementario de trabajo de riesgo (SCTR), aunque debe notarse que la frecuencia de los procesos debidos al seguro obligatorio de accidentes de tránsito (SOAT) es mayor en los comenzados por las IAFAS.

La actual legislación pretende consolidar las facultades $y$ el posicionamiento de SUSALUD a nivel nacional, y concomitantemente, refuerza la idea del CECONAR como líder en MASC, posicionándolo como administrador del SENACOAS ${ }^{(25)}$. Estas disposiciones traen consigo la necesidad de reformar la base normativa del CECONAR, de modo que se adecue a las competencias otorgadas y se tenga la base para el desarrollo del SENACOAS. Ante ello, se requiere llevar a cabo la reformulación del estatuto, y la construcción de la regulación del SENACOAS, así como expandir a nivel nacional los servicios de conciliación y arbitraje que se ofrecen. Por consiguiente, se reconoce la necesidad de extensión de los servicios, la consolidación y eficiencia de CECONAR, lo cual se acompaña indudablemente del fortalecimiento de sus competencias y la especialización de sus recursos humanos. En virtud a lo expuesto, CECONAR desarrolla dos vías de expansión principales, así como una iniciativa de apoyo financiero para acrecentar el acceso por parte de los ciudadanos.

La primera consiste en una alianza con el Ministerio de Justicia y Derechos Humanos (MINJUS). Por ello, en junio de 2015 se celebró un convenio de colaboración interinstitucional que permite brindar el servicio gratuito de conciliación en salud en los 84 centros de conciliación a nivel nacional que tiene el MINJUS, así como, realizar la difusión efectiva de este servicio y de lo ofertado por CECONAR. Por su parte, CECONAR capacita a los conciliadores del MINJUS en conciliación especializada en salud, para cuyo efecto se elaboraron manuales y se desarrolló un taller de capacitación con horas presenciales y virtuales para los conciliadores del citado ministerio, en las regiones de la Intendencia Macrorregional Norte (IMRN): Tumbes, Piura, Lambayeque, Trujillo, Amazonas y Cajamarca. En la actualidad, estos 15 profesionales proporcionan servicios de conciliación especializada en salud.

El segundo canal de expansión se da a través de la creación de oficinas desconcentradas en las intendencias macrorregionales (IMR) y jefaturas zonales que SUSALUD tiene proyectado crear en el país, sin perjuicio de abrir oficinas en alianzas con los gobiernos regionales y locales. Al respecto, a finales de abril de 2016 se iniciaron las operaciones de la oficina desconcentrada de la IMRN: CECONAR Norte. Esta dependencia es un centro de conciliación autorizado 
Tabla 1. Distribución de frecuencias de los procesos arbitrales de acuerdo a la fuente de inicio (2011-2015)

\begin{tabular}{|c|c|c|c|c|}
\hline \multirow{2}{*}{ Año } & SCTR & Salud & SOAT & Total \\
\hline & $\mathbf{N}(\%)$ & $\mathbf{N}(\%)$ & $\mathbf{N}(\%)$ & $\mathbf{N}(\%)$ \\
\hline \multicolumn{5}{|c|}{ Arbitrajes iniciados por las IAFAS } \\
\hline 2011 & $19(90,5)$ & $0(0,0)$ & $2(9,5)$ & $21(9,2)$ \\
\hline 2012 & $22(91,7)$ & $2(8,3)$ & $0(0,0)$ & $24(10,5)$ \\
\hline 2013 & $24(96,0)$ & $1(4,0)$ & $0(0,0)$ & $25(11,0)$ \\
\hline 2014 & $26(89,7)$ & $2(6,9)$ & $1(3,4)$ & $29(12,7)$ \\
\hline 2015 & $55(42,6)$ & $6(4,7)$ & $68(52,7)$ & $129(56,6)$ \\
\hline Total & $146(64,0)$ & $11(4,8)$ & $71(31,1)$ & $228(100)$ \\
\hline \multicolumn{5}{|c|}{ Arbitrajes iniciados por asegurado/beneficiario } \\
\hline 2011 & $241(96,0)$ & $10(4,0)$ & $0(0,0)$ & $251(21,3)$ \\
\hline 2012 & $235(94,8)$ & $13(5,2)$ & $0(0,0)$ & $248(21,0)$ \\
\hline 2013 & $182(94,8)$ & $9(4,7)$ & $1(0,5)$ & $192(16,3)$ \\
\hline 2014 & $238(90,5)$ & $25(9,5)$ & $0(0,0)$ & $263(22,3)$ \\
\hline 2015 & $220(96,9)$ & $7(3,1)$ & $0(0,0)$ & $227(19,2)$ \\
\hline Total & $1116(94,5)$ & $64(5,4)$ & $1(0,1)$ & $1181(100)$ \\
\hline
\end{tabular}

SCTR: Seguro Complementario de Trabajo Riesgo.

SOAT: Seguro Obligatorio de Accidentes de Tránsito.

IAFAS: instituciones administradoras de fondos de aseguramiento en Salud

por el MINJUS, donde se tramitan gratuitamente las solicitudes de conciliación, y otras peticiones que requieren resolver controversias en salud, que debido al incumplimiento de los requisitos de la conciliación, son atendidas vía IBOS.

Una tercera vía por desarrollar, es la incorporación de centros de conciliación y arbitraje privados, que cumpliendo con los estándares que se establezcan en el marco del SENACOAS, brinden servicios de conciliación y arbitraje especializado en salud a costos razonables, ampliando aun más la oferta de servicios de solución de controversias por medios alternativos.

Adicionalmente, con la voluntad de eliminar una de las principales barreras de acceso a la conciliación especializada en salud, CECONAR ha incorporado la gratuidad total del servicio de conciliación, ello implica la eliminación de los costos administrativos y del conciliador. Esta medida implementada desde el 2016 ha permitido que los usuarios de los servicios de salud accedan con menos restricciones al citado mecanismo alternativo. Al respecto, se ha observado que el número de solicitudes de conciliación en el primer cuatrimestre del año 2016 se ha triplicado en relación a la frecuencia de estas en un similar periodo durante el año 2015.

\section{DISCUSIÓN Y PERSPECTIVAS}

CECONAR es un centro de conciliación que opera bajo las normas de la Ley de Conciliación Extrajudicial (10,11), cuya rectoría la ejerce el MINJUS. A su vez, es un centro de arbitraje especializado en salud, bajo la regulación de la Ley de Arbitraje ${ }^{(26)}$. En este sentido, los servicios que brinda son de conciliación extrajudicial especializada en salud, arbitraje, bajo el tipo de arbitraje institucional, y mediación vía IBOS, y se accede a ellos de manera indistinta o alternativa, por voluntad del interesado por mandato legal en el caso del arbitraje sobre controversias derivadas del SCTR.

Con el AUS, CECONAR se amplió el ámbito de las controversias sobre las cuales podía ocuparse, incorporando además de los rembolsos por atenciones médicas, planes de cobertura, menoscabo para determinar la discapacidad de los accidentes o enfermedades profesionales, discrepancias contractuales entre la Seguridad Social en Salud (EsSalud) y las EPS, a todas aquellas controversias que surjan del intercambio prestacional entre los actores del aseguramiento, incluyendo los daños por mala práctica profesional o responsabilidad institucional que se presenten en todos los servicios de salud, y ya no limitado a las EPS.

En América Latina, los medios alternativos de solución de controversias se encuentran ampliamente desarrollados y en todos se tiene con legislación específica, aunque no respecto de todos los MASC (Tabla 2). No obstante, debemos indicar que el uso de los MASC incorporados específicamente en una norma legal con un ente especializado como en el caso peruano, no tiene homólogo en la región. La experiencia más cercana es la Comisión Nacional de Arbitraje Médico de México (CONAMED) creado en 1996, y destinada a brindar servicios de conciliación y arbitraje en casos de mala práctica, bajo un esquema progresivo y voluntario, en el cual cuando las partes no alcanzan un acuerdo en la conciliación, se 
Tabla 2. Breve descripción de las experiencias relacionadas a conciliación y arbitraje en países en América Latina

\begin{tabular}{|c|c|c|c|}
\hline Países & Base Legal & Modalidad & Observaciones \\
\hline Argentina & $\begin{array}{l}\text { Ley } 24573 \text {, Ley de Mediación para } \\
\text { Materias Civiles y Comerciales, } \\
\text { modificada por la Ley } 26589 . .^{*}\end{array}$ & $\begin{array}{l}\text { La mediación es obligatoria y } \\
\text { el arbitraje es voluntario. }\end{array}$ & $\begin{array}{l}\text { No se encuentra regulada la solución } \\
\text { de controversias en materia de salud } \\
\text { a través de MASC. }\end{array}$ \\
\hline Bolivia & $\begin{array}{l}\text { Ley 708, Ley De Conciliación y } \\
\text { Arbitraje.* }\end{array}$ & $\begin{array}{l}\text { La conciliación extrajudicial y } \\
\text { el arbitraje son voluntarios. }\end{array}$ & $\begin{array}{l}\text { No se encuentra regulada la solución } \\
\text { de controversias en materia de salud } \\
\text { a través de MASC. }\end{array}$ \\
\hline Brasil & $\begin{array}{l}\text { Ley 9307, Ley de Arbitraje Comercial e } \\
\text { Internacional. } \neq\end{array}$ & $\begin{array}{l}\text { La mediación, conciliación } \\
\text { extrajudicial y el arbitraje son } \\
\text { voluntarios. }\end{array}$ & $\begin{array}{l}\text { No se encuentra regulada la solución } \\
\text { de controversias en materia de salud } \\
\text { a través de MASC. }\end{array}$ \\
\hline Chile & $\begin{array}{l}\text { Ley 19971, Ley de Arbitraje Comercial } \\
\text { Internacional. } \\
\text { Ley de Mediación en Salud } 19966 . \\
\text { Ley de Mediación Familiar } 20286 .\end{array}$ & $\begin{array}{l}\text { La mediación es obligatoria, } \\
\text { la conciliación es parte del } \\
\text { proceso judicial, el arbitraje es } \\
\text { voluntario. }\end{array}$ & $\begin{array}{l}\text { La Superintendencia de Salud, } \\
\text { resuelve controversias entre las } \\
\text { instituciones de salud previsional o } \\
\text { el Fondo Nacional de Salud y sus } \\
\text { cotizantes o beneficiarios }{ }^{(28)} \text {. }\end{array}$ \\
\hline Colombia & $\begin{array}{l}\text { Ley } 446 \text { de 1998, Desarrolla los } \\
\text { Mecanismos Alternativos de Solución de } \\
\text { Conflictos. } \\
\text { Decreto 1818, Estatuto de los } \\
\text { Mecanismos Alternativos de Solución de } \\
\text { Conflictos. } \\
\text { Ley } 1563 \text { del 2012, Estatuto de Arbitraje } \\
\text { Nacional e Internacional. }\end{array}$ & $\begin{array}{l}\text { La conciliación es obligatoria y } \\
\text { el arbitraje es voluntario. }\end{array}$ & $\begin{array}{l}\text { No se encuentra regulada la solución } \\
\text { de controversias en materia de salud } \\
\text { a través de MASC. }\end{array}$ \\
\hline Ecuador & $\begin{array}{l}\text { Ley } 145, \text { Ley de Arbitraje y Mediación } \\
1997^{*}\end{array}$ & $\begin{array}{l}\text { Voluntario La mediación y el } \\
\text { arbitraje son voluntarios. }\end{array}$ & $\begin{array}{l}\text { No se encuentra regulada la solución } \\
\text { de controversias en materia de salud } \\
\text { a través de MASC. }\end{array}$ \\
\hline Paraguay & Ley 1879, Ley de Arbitraje y Mediación * & $\begin{array}{l}{ }^{4 /} \text { Voluntario La mediación y el } \\
\text { arbitraje son voluntarios. }\end{array}$ & $\begin{array}{l}\text { No se encuentra regulada la solución } \\
\text { de controversias en materia de salud } \\
\text { a través de MASC. }\end{array}$ \\
\hline Perú & $\begin{array}{l}\text { Ley 26872, Ley de Conciliación y } \\
\text { Decreto Legislativo } 1070 \dagger \\
3 / \text { Decreto Legislativo } 1071 \text {, Decreto } \\
\text { Legislativo que norma el arbitraje. } \ddagger\end{array}$ & $\begin{array}{l}\text { La conciliación extrajudicial } \\
\text { es obligatoria y el arbitraje es } \\
\text { voluntario. }\end{array}$ & $\begin{array}{l}\text { Centro de Conciliación y Arbitraje } \\
\text { - Superintendencia Nacional de } \\
\text { Salud, procura la solución de las } \\
\text { controversias en la prestación de } \\
\text { servicios de salud y demás derechos } \\
\text { relacionados, principalmente en el } \\
\text { ámbito del AUS y la Seguridad Social. }\end{array}$ \\
\hline Uruguay & $\begin{array}{l}\text { Ley } 15.982 \text {, Código General del } \\
\text { Proceso, modificada por Ley } 16.995, \\
\text { Conciliación previa a los juicios }\end{array}$ & $\begin{array}{l}\text { El arbitraje es voluntario y } \\
\text { conciliación obligatoria. }\end{array}$ & $\begin{array}{l}\text { No se encuentra regulada la solución } \\
\text { de controversias en materia de salud } \\
\text { a través de MASC. }\end{array}$ \\
\hline Venezuela & Ley de Arbitraje Comercial de 1998 ‡ & El arbitraje es voluntario. & $\begin{array}{l}\text { No se encuentra regulada la solución } \\
\text { de controversias en materia de salud } \\
\text { a través de MASC. }\end{array}$ \\
\hline México & $\begin{array}{l}\text { Ley de Mediación, Conciliación y } \\
\text { Promoción de la Paz Social para el } \\
\text { Estado de México - Decreto 251. } \\
\text { Código de Comercio de 1993: Título } \\
\text { Cuarto. El Arbitraje Comercial. }\end{array}$ & $\begin{array}{l}\text { Mediación y conciliación } \\
\text { son voluntarios, arbitraje } \\
\text { voluntario. }\end{array}$ & $\begin{array}{l}\text { Comisión de Conciliación y Arbitraje } \\
\text { Médico - Secretaría de Salud } \\
\text { (CCAMEM), resuelve conflictos entre } \\
\text { usuarios y prestadores de salud, sean } \\
\text { éstos públicos, privados o mixtos }{ }^{(27)} \text {. }\end{array}$ \\
\hline
\end{tabular}

* Normativa para mediación, conciliación y arbitraje.

†Normativa para mediación y conciliación.

† Normativa para arbitraje.

MACS: mecanismos alternativos de solución de controversias

procede al arbitraje ${ }^{(27)}$. Otra experiencia cercana es la chilena, en razón a la obligatoriedad de acudir a un MASC, previa a la vía ordinaria, aunque solo está circunscrito a las controversias cuyo origen es la mala práctica y no otros conexos como en el caso peruano. La ley en Chile exige a quien pretende iniciar un proceso judicial por mala práctica, previamente acudir a la vía de mediación en salud ${ }^{(28)}$.
CECONAR se encuentra en franco desarrollo y extensión de sus servicios de conciliación extrajudicial especializada en salud a nivel nacional, soportado por los centros de conciliación del MINJUS y sus propias oficinas desconcentradas. Es importante que el énfasis de la extensión de sus servicios se concentre en aquellas provincias donde la obligatoriedad de la conciliación 


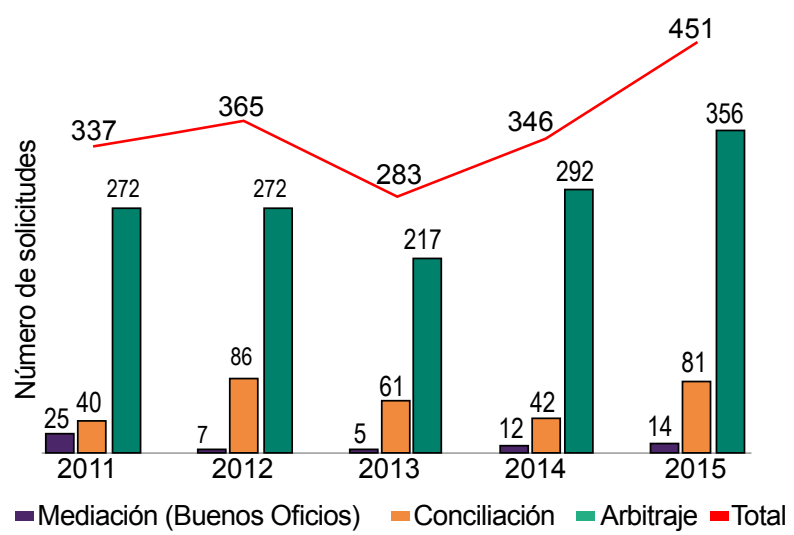

Figura 1. Distribución de frecuencias de solicitudes de mediación, conciliaciòn y arbitraje atendidos por CECONAR (2011-2015)

ya ha sido instaurada por el MINJUS. Ello contribuirá notablemente al cumplimiento del mandato legal de la especialidad -contenido en el Decreto Legislativo $1158^{(25)}$ - y la obligatoriedad del intento conciliatorio que establece la Ley de Conciliación Extrajudicial (10,11), creando oportunidades de solución de controversias. No obstante, debe precisarse que para lograr la ejecución plena de lo pactado con el MINJUS y contar con los servicios de conciliación de sus 84 centros, se requiere capacitar a 89 conciliadores de dicho ministerio, quienes ejercen en regiones distintas a la jurisdicción de la IMRN, lo cual supone una importante inversión económica.

Asimismo, CECONAR debe concebir y elaborar los criterios y parámetros que permitan registrar y habilitar a los centros de conciliación extrajudicial y a los centros de arbitraje privados especializados en salud, así como a los árbitros y conciliadores que desarrollen sus servicios en dichos centros, lo que exige establecer estándares para los centros, y se enlaza ineludiblemente con el diseño de los perfiles para sus conciliadores y árbitros. El cumplimiento de las exigencias planteadas permitirá su adscripción al SENACOAS, con lo que se incrementaría la oferta de servicios de solución alternativa de controversias. Claro está que lo propuesto debe acompañarse de las modificaciones legales en cuanto a la supervisión, fiscalización y sanción por incumplimiento de los criterios y parámetros que se establezcan, así como regular lo relativo a la dirimencia. Todo ello, en cuanto a la conciliación se refiere, debe ser coordinado con el MINJUS, en su condición de ente rector de la conciliación extrajudicial en el País.

Adicionalmente, como ya se ha expuesto, los servicios de CECONAR en materia arbitral demandan mejoras substanciales en términos de eficiencia, para ello se ha concebido un nuevo proceso arbitral más ágil y la incorporación de herramientas tecnológicas con el propósito de optimizar los tiempos y reducir los costos asociados. Dentro de las innovaciones propuestas se encuentra la notificación electrónica, el desarrollo de audiencias virtuales y la digitalización del expediente arbitral sobre la base del sistema de atención al usuario existente en CECONAR. Igualmente, se planea el desarrollo de un portal web y una App que permitirán un fácil acceso a los usuarios para la revisión del estado de su expediente, así como la recepción de notificaciones en tiempo real de su proceso arbitral, tanto desde la computadora como desde un celular. Sin embargo, es importante remarcar que en materia de conciliación, la rigidez de la normativa vigente y de la rectoría del MINJUS, no permiten introducir variaciones en su procedimiento ni establecer notificaciones electrónicas.

\section{CONCLUSIONES}

En Perú, CECONAR lidera los MASC, y en dicho contexto, tanto la mediación, la conciliación y el arbitraje constituyen procesos válidos y accesibles para todos los actores del SSP. Se reconoce que este accionar contribuye a la consolidación del ciudadano como eje central en el marco de los DES. No obstante, se requiere un abordaje multidisciplinario, un trabajo sinérgico con otras dependencias de SUSALUD, una labor cooperativa con otras instituciones y un importante soporte financiero, en pos de mantener su funcionamiento, y asumir positivamente los retos que su expansión y consolidación demandan. Las acciones y estrategias aludidas vislumbran un futuro prometedor para los MASC en el país.

Contribuciones de autoría: DHS, COP, EMH realizaron contribuciones substanciales en la concepción del artículo. DHS, COP, EMH realizaron contribuciones substanciales en el diseño del artículo. JLB, PHN, EMH, realizaron la adquisición de datos. JLF, PHN, EMH, realizaron el análisis de datos. DHS, JLF, PHN interpretaron los de datos. DHS, COP, EMH, redactaron el primer borrador del artículo. DHS, JLF, PHN realizaron una revisión crítica que proporciona un importante contenido intelectual. Todos los autores aprobaron la versión final del artículo y están de acuerdo en ser responsables de todos los aspectos relacionados al artículo, lo que implica asegurar de que todas las preguntas relacionadas a la validez e integridad de cualquier parte del artículo han sido apropiadamente investigadas y resueltas.

Fuente de financiamiento: el presente artículo fue financiado por la Superintendencia Nacional de Salud (SUSALUD)

Conflictos de interés: al momento de la publicación los autores laboran en la Superintendencia Nacional de Salud (SUSALUD). No obstante, este documento no representa un posicionamiento oficial por parte de SUSALUD. 


\section{REFERENCIAS BIBLIOGRÁFICAS}

1 Gruskin S, Mills EJ, Tarantola D. History, principles, and practice of health and human rights. Lancet. 2007;370(9585):449-55.

2 Eleftheriadis P. A right to health care. J Law Med Ethics. 2012;40(2):26885 . doi: $10.1111 /$ j.1748720X.2012.00663.x.

3 Landa C, Velasco A. Constitución política del Perú 1993: Sumillas, Reformas Constitucionales, Índice Analítico. $7^{\mathrm{a}}$ ed. Lima: Fondo Editorial Pontifica Universidad Católica del Perú; 2007.

4 Perú, Ministerio de Salud. Decreto Supremo 027-2015-SA. Aprueban Reglamento de la Ley que establece los Derechos de las Personas Usuarias de los Servicios de Salud. (13 de agosto de 2015).

5 Bergman EJ. Identifying Sources of Clinical Conflict: A Tool for Practice and Training in Bioethics Mediation. J Clin Ethics. 2015;26(4):315-23.

6 Kelly J. An overview of conflict. Dimens Crit Care Nurs. 2006;25(1):22-8.

7 Tuya-Figueroa X, Mezones-Holguín E. Violencia contra médicos: un problema por considerar en la investigación de recursos humanos en salud. Rev Peru Med Exp Salud Publica. 2012;29(1):164-5.

8 Alcalde-Rabanal JE, Lazo-González O, Nigenda G. Sistema de salud de Perú. Salud Publica Mex. 2011;53 Suppl 2:S243-54.

9 Cantuarias-Salaverry F, Roque J Caivano. La Nueva Ley de Arbitraje Peruana: Un nuevo salto a la modernidad. Rev Peru Arbitr. 2008;7:43-84.

10 Perú, Congreso de la Republica. Ley 26872, de Conciliación Extrajudicial. (29 de octubre de 1996).

11 Decreto Legislativo 1070. Decreto que Modifica la Ley 26872, Ley de Conciliación. (21 de junio 2008).

12 Bustamante-Leija LE, MaldonadoCamargo VM, González-Anaya
C, Gutiérrez-Vega R. Mecanismos alternativos de solución de controversias en la prestación de servicios de salud. Rev CONAMED. 2016;17(3):126-9.

13 Holbrook JR. Negotiating, mediating, and arbitrating physician-patient conflicts. Clin Obstet Gynecol. 2008;51(4):719-30. doi: 10.1097/ GRF.0b013e3181899cf9.

14 Gonzalo-Quiroga M, Francisco Gorjón-Gómez, Sánchez-García A. Métodos alternativos de solución de conflictos: perspectiva multidisciplinar: Herramientas de paz y modernización de la justicia. Madrid: DYKINSON, S.L.; 2011

15 Caivano RJ, Gobbi M, Padilla RE. Negociación y mediación: instrumentos apropiados para la abogacía moderna. Buenos Aires: Ad-Hoc; 1997.

16 Quiroga-León A. Conciliación y Arbitraje en el Perú: presente y futuro. Derecho PUCP. 2013;53:769-98.

17 Juárez-Jurado E. Constitucionalización y control constitucional del arbitraje en el Estado Constitucional [Tesis magister]. Lima: Pontificia Universidad Católica del Perú; 2015.

18 Perú, Congreso de la República. Ley 26790. Ley de Modernización de la Seguridad Social en Salud. (17 mayo de 1997).

19 Perú, Ministerio de Salud. Decreto Supremo 009-97-SA. Aprueban Reglamento dela Ley de Modernización de la Seguridad Social en Salud. (8 de septiembre de 1997).

20 Perú, Ministerio de Salud. Decreto Supremo 006-97-SA. Aprueban el Estatuto de la Superintendencia de Entidades Prestadoras de Salud. (25 de junio de 1997).

21 Perú, Ministerio de Salud. Decreto Supremo 005-98-SA. Aprueban el Reglamento de Organización y Funciones de la Superintendencia de Entidades Prestadoras de Salud. (5 de junio de 1998).
22 Perú, Ministerio de Salud. Ley Marco de Aseguramiento Universal en Salud. Ley 29344. (30 de marzo de 2009).

23 Wilson L, Velásquez A, Ponce C. La ley marco de aseguramiento universal en salud en el Perú: análisis de beneficios y sistematización del proceso desde su concepción hasta su promulgación. Rev Peru Med Exp Salud Publica. 2009;26(2):207-17.

24 Perú. Ministerio de Salud. Decreto Supremo 008-2010-SA. Aprueban Reglamento de la Ley marco de Aseguramiento Universal en Salud. (3 abril de 2010).

25 Perú, Presidencia de la República. Decreto Legislativo 1158. Decreto Legislativo que dispone de medidas destinadas al fortalecimiento y cambio de denominación de la Superintendencia Nacional de Aseguramiento en Salud. (6 diciembre de 2013).

26 Perú, Presidencia de la República. Decreto Legislativo 1071. Decreto Legislativo que norma el Arbitraje. (1 de septiembre de 2008)

27. Varela HF. La comisión nacional de arbitraje médico. Rev Fac Med. 2000;43(4) 151-2.

28. Mera A. Mecanismos alternativos de solución de conflictos en América Latina. diagnóstico y debate en un contexto de reformas. En: Aportes para un diálogo sobre el acceso a la justicia y reforma civil en América Latina. Santiago: Centro de Estudio de Justicia de las Américas; 2013. p. 375-433.

Correspondencia: Edward Mezones-Holguin Dirección: Av. Velasco Astete 1398, Santiago de Surco. Lima, Perú.

Teléfono: (+511) 3726150 anexo 5412

Correo electrónico: emezones@gmail.com / emezones@susalud.gob.pe 